Document downloaded from:

http://hdl.handle.net/10251/45040

This paper must be cited as:

Sendra Compte, S.; Llario Sempere, F.; Parra Boronat, L.; Lloret, J. (2013). Smart Wireless Sensor Network to Detect and Protect Sheep and Goats to Wolf Attacks. Recent Advances in Communications and Networking Technology. 2(2):91-101. doi:10.2174/22117407112016660012.

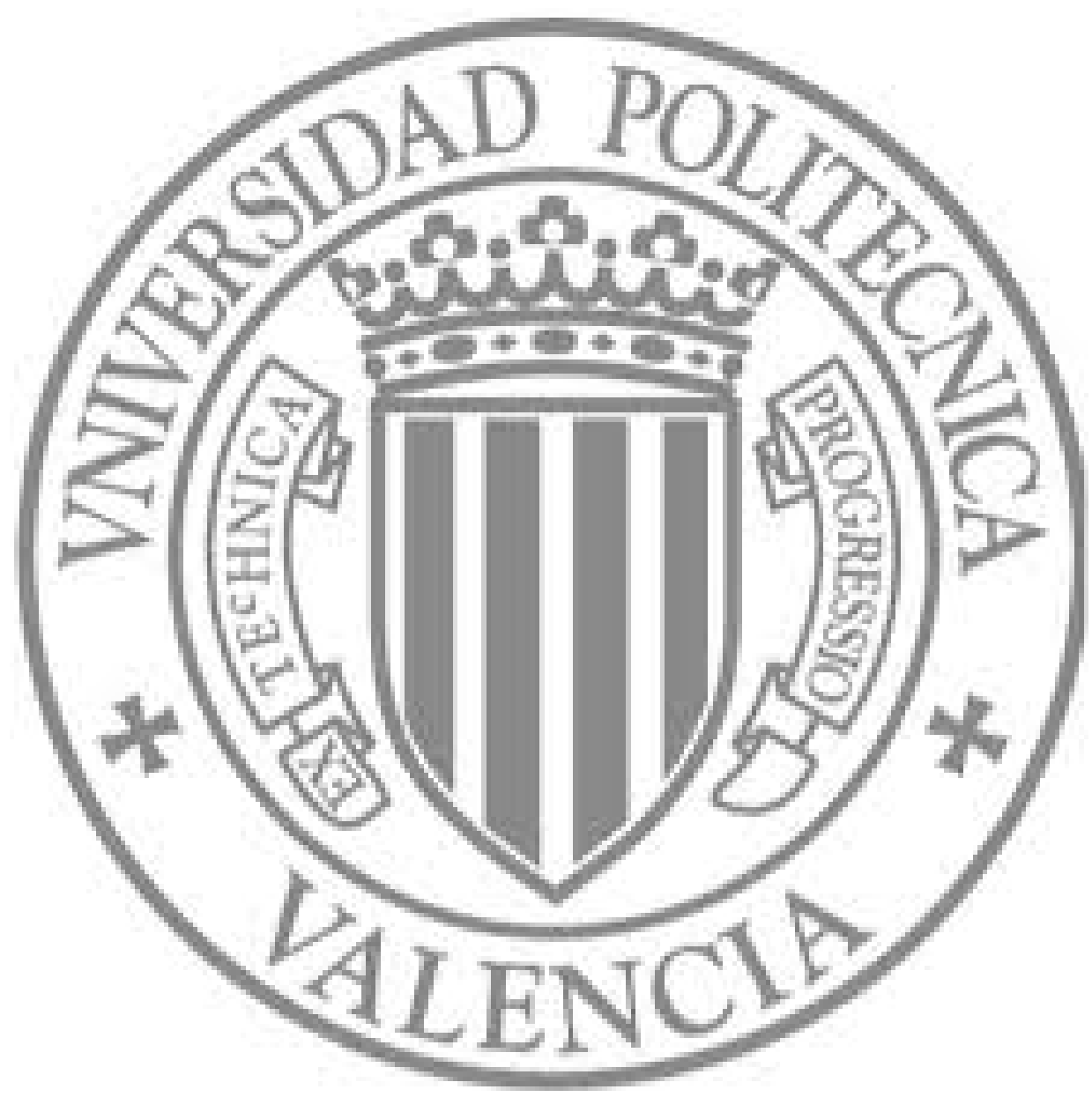

The final publication is available at

http://dx.doi.org/10.2174/22117407112016660012

Copyright Bentham Science Publishers 


\title{
Smart Wireless Sensor Network to Detect and Protect Sheep and Goats to Wolf Attacks
}

\author{
Sandra Sendra, Ferran Llario, Lorena Parra, Jaime Lloret \\ Universidad Politécnica de Valencia \\ Camino Vera s/n, 46022, Valencia, Spain \\ sansenco@posgrado.upv.es, ferllase@epsg.upv.es, loparbo@epsg.upv.es, jlloret@dcom.upv.es
}

\begin{abstract}
The attacks on flocks of goats and sheep are causing great economic losses in some countries. The main causes of these are usually wild animals such as wolves, or thefts of young animals. When an animal feels the presence of people or animals different than the usual ones, it experiences changes in its vital signs. The parameters which are the most altered are the heart rate and body temperature. This paper presents the development of wireless sensor nodes which can monitor vital signs of sheep and goats. These nodes form a network of wireless nodes using IEEE 802.15.4 technology. The network runs a smart algorithm able to detect episodes of collective stress on flocks of goats and sheep caused by any predator attack overnight. When the system detects changes in vital signs of animals, it sends an alarm signal to alert the person in charge of the livestock facility and activates audible and visual alarms able to scare off predators and marauders. Our system can help breeders of goats and sheep to protect their herds avoiding large economic losses that this implies.
\end{abstract}

Keywords- IEEE 802.15.4, Electronic deployment, Wireless sensor network, Smart detection system, sheep and goats stress, wolves attacks.

\section{INTRODUCTION}

A Wireless Sensor Network (WSN) can be defined as a network of small embedded devices, called sensors which communicate wirelessly. They are located strategically inside a physical medium and are able to interact with it in order to measure physical parameters from the environment and provide the sensed information. These nodes can register variations in the medium, thanks to the physical transducers, and transmit this information to the network. The nodes usually are small size devices in order avoid causing any visual impact to the ecosystem and they should not consume too much energy. This is one of the main problem which is being investigated [1]. We should also keep in mind that the nodes have limited data process and storage capacity.

Nowadays, the use of WSNs is spreading more and more in rural areas. This happens because WSNs allow environmental monitoring and the management of several activities in agricultural and rural areas [2][3]. In addition, the infrastructure needed for this task is simple while it has quite low economical costs and other benefits compared to other infrastructures [4].

WSNs can be used in several activities in the agricultural sector where the combination of new technologies and the knowledge of some existing problems can provide many benefits. In the livestock sector, a WSN could be used for controlling the animals and other environmental parameters, such as attempts of animals' theft or flock attacks by wild animals, among others.

In 2011, the "Asociación Agraria de Jovenes Agricultores" (ASAJA) [5], announced that the number of wolf attacks (Canis lupus) in Castilla y Leon (Spain) had increased up to $42 \%$ with 2,400 dead sheep and economical losses near to 335,000 €. In the same year, the Principado de Asturias' (Spain) government announced that the number of wolfs attacks had stabilized at an average of 3,500 attacks per year (after an increase of 24\% of these attacks during 2008 and 2009) [6].

Wolf attacks are also continuously growing in France, reaching values of 4,290 victims in 2011. As Figure 1 shows, these livestock losses were translated into economic losses of 1,548,052 Euros to the French government [7]. These attacks are registered mainly in the north of Spain and in most of the pastoral areas of the rest of Europe. Massive wolf attacks cause the illegal hunting of these predators and the incompatibility of the wolf recovery (because this species is considered endangered). Furthermore, the financial resources designed for rural areas are also negatively affected.

This paper is an extended version of the paper presented in the "3rd IEEE International Workshop on Smart Communication Protocols and Algorithms" [8] where we proposed the use of an intelligent system based on an IEEE 802.14.5 WSN to avoid and prevent wolf attacks and thefts. We did not provide any detail about a possible deployment.

In this paper, we present the development of a WSN capable to intelligently detect when a flock is being attacked by wild animals. We also present the deployment of all circuits needed to implement our system. Furthermore, this paper provides the simulations of circuits to check the correct operation of them. Each node provides the data of temperature and cardiac frequency of each animal to the server which is in charge of processing all data. From these data and using the intelligent algorithm the server will determine if the flock is being attacked or not. The server has the main capacity of processing and it 
will define what kind of alarm has to be generated and consequently, the system will warn the owner and other services such as the environmental unit of animal control.

We could think that these attacks can be detected using an intruder detection system deployed on the barrier. However, our sheeps and goats could active the alarm generating a false positive. Our system uses an intelligent algorithm to store and tagging the kinds of alarms generated along the time.

The rest of this paper is structured as follows. Section 2 shows several related works where sensors or sensor networks are used for monitoring parameters in livestock and agriculture sector. Section 3 shows the circuits and operation of our circuits and the placement of sensors on the sheep. Section 4 explains the operation of the wireless sensor network, its infrastructure and the alarm system. The protocol and smart algorithms designed and developed for the proper operation of our system are presented in section 5. They are able to send the appropriate alarm in each case by using a learning phase. Section 6 shows the performance of the developed system. Finally, Section 7 draws the conclusions and analyzes the designed system.

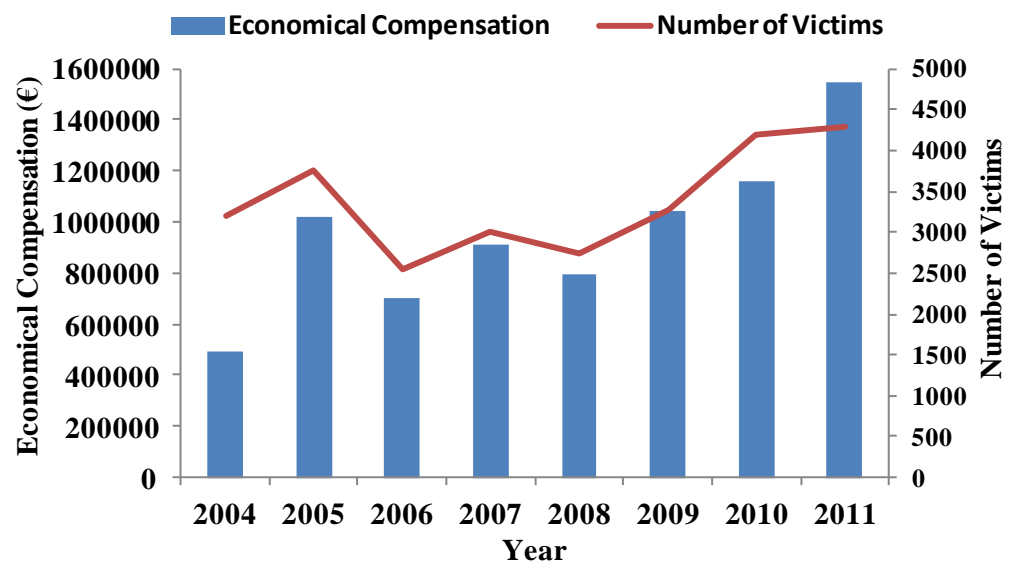

Figure 1. Number of wolves attacks to flocks in France

\section{RELATED WORKS}

Nowadays, new technologies are widely used in livestock and agriculture [9] for health monitoring and for animal identification [10]. This section presents some of the main works where WSN are applied to solve problems in livestock and agriculture sectors.

In [11], Lloret et al. presented WSN based on a image processing system for Vineyard Monitoring. This system is able to detect if a vine has bad leaves or not. When the wireless sensor detects any unusual status in the vine leaves, it generates an alarm that will be routed to the sink node in order to warn the farmer.

Kwong et al. propose [12] the adaptation of a WSN for cattle monitoring application. Authors show a solution for animal monitoring system by using low-cost, low power consumption sensor nodes. Their system is based on a collar formed by two antennae and several sensors to monitor vital parameters of animals. Nodes use the IEEE 802.15.4 technology. To facilitate real-time reporting, authors designed an Implicit Routing Protocol (IRP) which is implemented on the MICAz nodes using TinyOS sensor network operation system.

Sensor networks are used in extensive cattle for monitoring remotely the flock position in the area of livestock grazing [13]. In intensive farming, sensor nodes are used to control environmental variables, such as temperature or humidity [14].

Regarding to flock monitoring, we can use sensor networks to detect strange behavior in animals. These behaviors can be generated in several animals by presence of wild animals and strange persons. The stress in animals is usually related with variations of corporal temperature, heart rate and respiratory rate [15]. For this type of monitoring, it can be used under skin sensors [16, 17] or outer sensors [18]. The second method is less invasive for animals.

In [19], authors show an experiment where a sheep, in a regular situation, has heart rates between 60 to 80 beats per minute, meanwhile the animal is being attacked or stressed by other causes, it can register heart frequencies up to 225 beats per minute. Finally, the biologist and inventor of this prototype Jean-Marc Landry aims to study a system that strews a chemical repellent on the wolf to generate its flee.

The monitoring of these parameters allows us the detection of collective stress episodes in sheep and goats flock caused by wolf attacks. So, if we are able to detect any sign of attack (by the animal behavior), we will able to generate acoustic and visual alarms in order to scare the wild animals, and avoid the attack.

As far we know, there is not smart system similar to our proposal. Our proposal is a non-invasive system able to provide real-time data about vital signs of animals. From these values, our system will decide if the flock is being attacked or not. 


\section{MONITORING SYSTEM OF HEART RATE AND BODY TEMPERATURE.}

In the presence of a threat as wolves or unknown persons, the sheep undergoes an elevation of heart rate and body temperature. At rest, the temperature of a sheep is around $38.5-39.5^{\circ} \mathrm{C}$. Sheep and goats have a resting heart rate around $70-$ 80 beats per minute. In a stressful situation, this value can be tripled. Sheep, as well as other mammals, have different points where the heart rate can be measured. To determine heart rate, we must measure it as a differential measurement of the values recorded in 2 or 3 different points of the body. The connections arrangement of each electrode group is called bypass. In our proposal, we use the limb leads and we are going to employ two measuring points on the body of the animal.

This section will show the proposed circuit for our belt monitoring system. For this measurement, it is proposed the use of an instrumentation amplifier followed by selective filters, to capture the signals correctly. Finally, we present the measurement system for the animal and the ON/OFF circuit to control the node.

With the aim of monitoring the sheep body temperature and its heart rate, we propose to use a platinum temperature sensor, which is in contact with the animal body. To measure heart rate, we will implement an ECG circuit using an instrumentation amplifier able to take differential measures. The electrodes will be connected to the front legs of the animal. Thus, it will determine the heart rate of the animal using DI limb bypass. DI is a connection between electrodes on the front left leg and the front right leg. When the front left leg is in a positive force field over the front right leg, in DI is detected a positive upward deflection.

Figure 2 shows the block diagram of the circuit. The entire circuit is located in a small box on the animal's collar. Each of these boxes has a wireless node based on IEEE 82.15.4 technology. The node is powered by a rechargeable battery. The measures from both the temperature sensor and ECG circuit will be processed by the node, which will establish a connection with the Zigbee AP. Recharging the battery is done through a flexible solar panel to be integrated into sheep collar. The regulator will be in charge to determine at what time the battery must be recharged or not. Finally, because the wolves attack occur at night, our node will monitor the animal during the hours of darkness. The rest of time, the node will remain off. Figure 3 shows the belt with sensors.

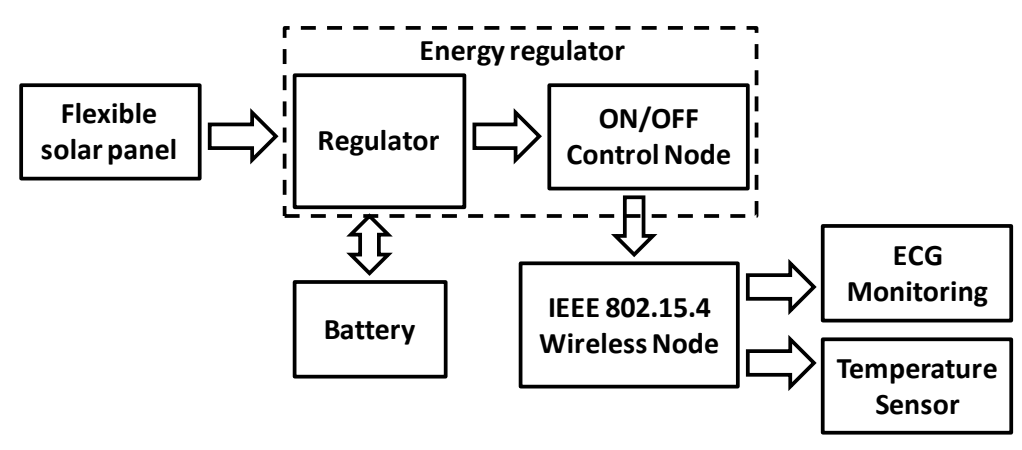

Figure 2. Circuit diagram to monitor the sheep.

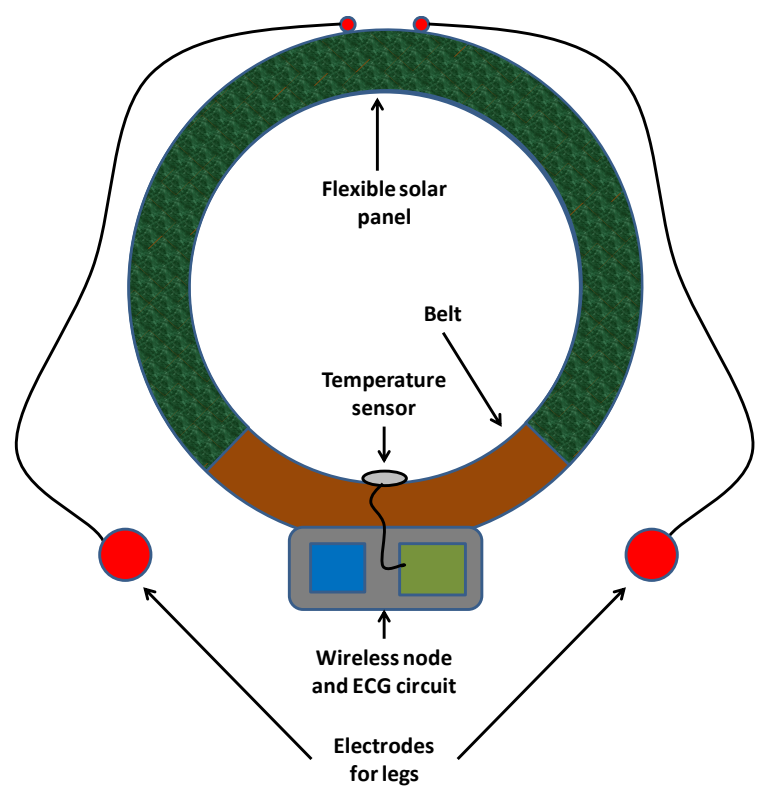

Figure 3. Belt whit sensors.

\subsection{Body temperature sensor}

To implement the sensor body temperature, we use a RTD [20] mounted within a Wheatstone bridge. This platinum sensor is able to measure the temperature in a range between $-200{ }^{\circ} \mathrm{C}$ and $+750{ }^{\circ} \mathrm{C}$ with high accuracy and stability. It has a metal chassis which allows a quick response (max. 7.5 seconds for air) by temperature changes. The Wheatstone bridge output is connected to an operational amplificator in differential configuration. Figure 4 shows the circuit diagram for monitoring the body temperature. The output voltage and body temperature are related by Equation (1).

$$
\text { Vout }=\frac{R_{5}}{R_{4}} \cdot \frac{\frac{R_{1}}{R_{2}}-\frac{R_{0}(1+\alpha(t-273))}{R_{3}}}{\left(1+\frac{R_{1}}{R_{4}}\right)\left(1+\frac{R_{0}(1+\alpha(t-273))}{R_{3}}\right)} \cdot V_{\text {in }}
$$


Figure 5 shows the resistive value of temperature sensor as a function of temperature. Using a value of resistors $R_{5}=33 \mathrm{~K} \Omega$ and $\mathrm{R}_{4}=1.2 \mathrm{~K} \Omega$, we obtain a value of gain equal to 27.5 . This places the output signal between $0.124 \mathrm{~V}$ (for value of temperature of $0^{\circ} \mathrm{C}$ ) to $4.85 \mathrm{~V}$ (for value of temperature of $40^{\circ} \mathrm{C}$ ). (See Figure 6)

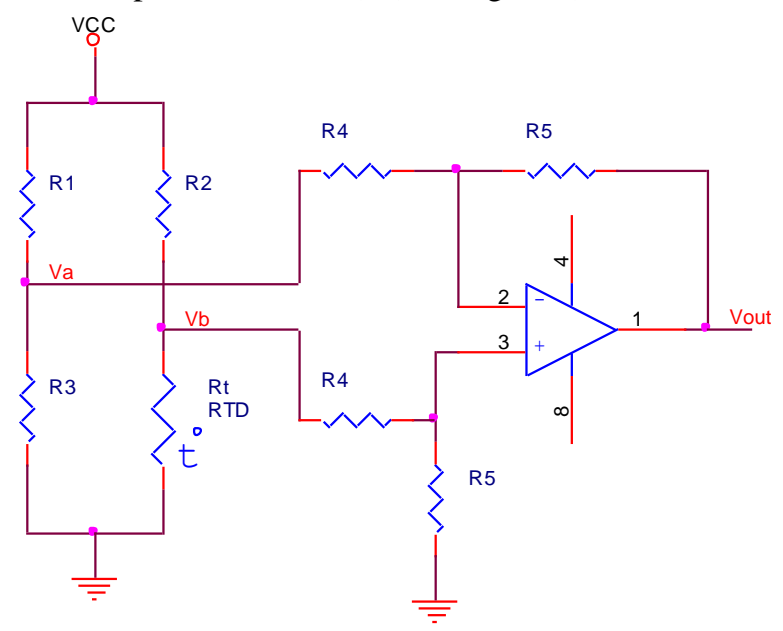

Figure 4. Temperature monitoring circuit

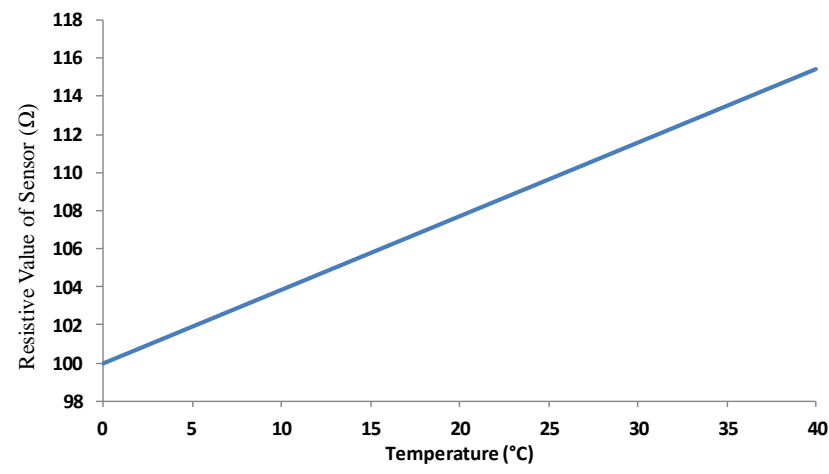

Figure 5. Resistive values as a function of temperature.

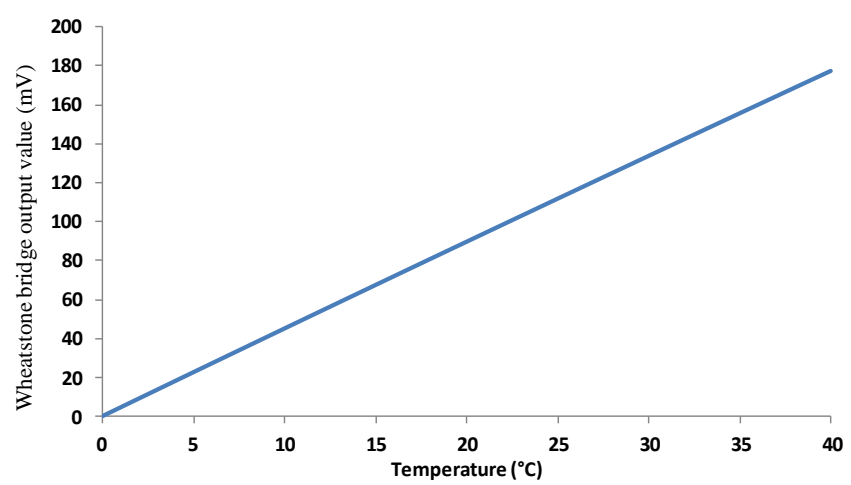

Figure 6. Output value of Wheatstone bridge as a function of temperature

\subsection{Cardiac frequency sensor.}

The ECG is a physical test widely used to assess, in a noninvasive way, the condition of the heart. This test can assess the state of the conduction system of the heart, its muscle, and indirectly, the condition of this organ. For the measurement of the sheep heart rate, we use a circuit based on three operational amplifiers of two stages. Figure 7 shows the schematic of our ECG circuit.

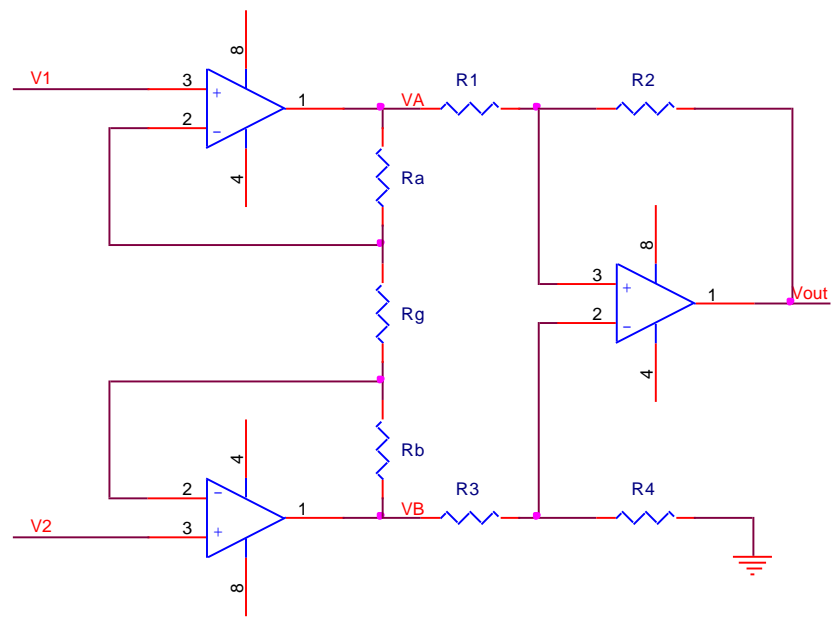

Figure 7. Circuit for cardiac frequency monitoring 


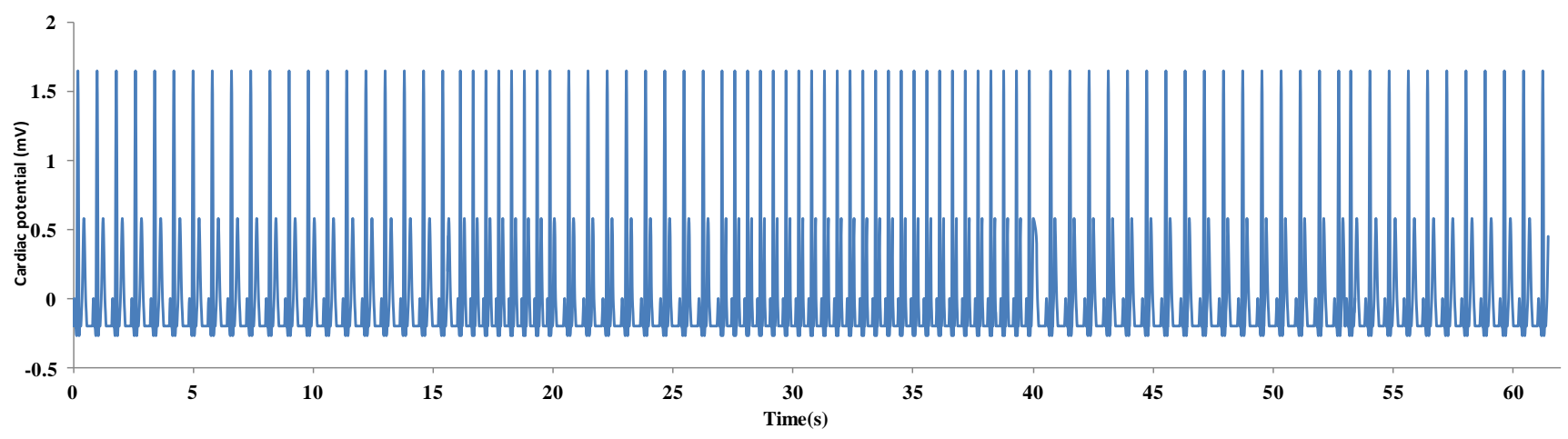

Figure 8. Signal of cardigram

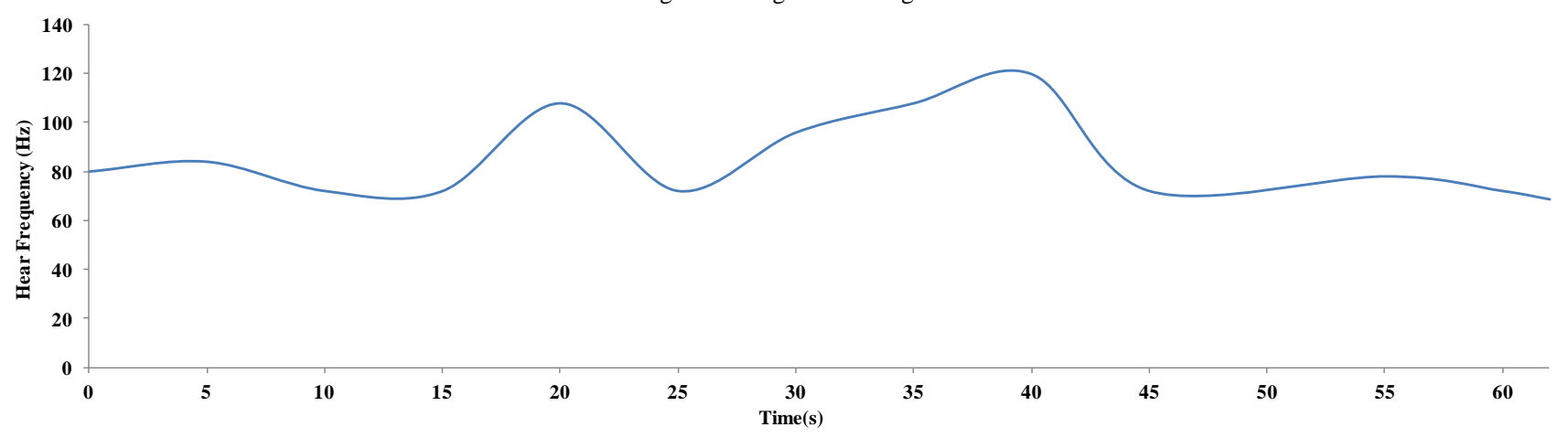

Figure 9. Cardiac Frequency obtained form cardiogram

Equation 2 shows the relationship between the output voltage (value to be processed) and voltage values taken in the right leg (V1) and the left leg (V2). The voltage values V1 and V2 are related to the frequency rate.

$$
\text { Vout }=\frac{\left(V_{A}-V_{B}\right)}{2} \cdot\left[\frac{1-\frac{R_{2} R_{3}}{R_{1} R_{4}}}{1-\frac{R_{3}}{R_{4}}}\right]-\left(V_{A}-V_{B}\right) \cdot\left[\frac{1-\frac{R_{2}}{R_{1}}}{1-\frac{R_{3}}{R_{4}}} \cdot\left(\frac{1}{2}+\frac{R_{b}}{R_{g}}\right)+\frac{R_{2}}{R_{1}} \cdot\left(\frac{1}{2}+\frac{R_{a}}{R_{g}}\right)\right]
$$

This circuit can be implemented with individual operational amplifiers or as an integrated designed for this purpose. In our case we will use the instrumentation operational amplifier INA 125 able to work in single supply. The amplifier gain can be adjusted using Equation 3.

$$
G=4+\frac{60 K \Omega}{R g}
$$

Figure 8 shows a cardiogram. As we can see, there is an increase of pulses between seconds 15 to 20 and 23 to 40 . It is easy to extract the value of cardiac frequency from a cardiogram shown in Figure 8. To do this, we have defined a perior of 5 seconds to estimate the average value of heart rate. Taking into account this value, Figure 9 shows the value of cardiac frequency.

\subsection{ON/OFF circuit to control the wireless node}

Wolves are animals of nocturnal habit. For this reason, our wireless nodes and consequently the monitoring of vital signs of sheep should operate overnight. To this end, we will employ a light control circuit which will act between the battery connection and the node. As a light sensor, we will use a light dependent resistor (LDR). The circuit operates as an electronic switch where the activation/deactivation of the relay and consequently the wireless node, depends solely on the bias of transistor Q1. The circuit is a simple bias network where the LDR controls the flow of current through the transistor base. Figure 10 shows the control circuit for wireless node where the relay will be activated in the presence of the dark.

\subsection{Placement of sensors on the animals}

Our circuits are protected by a small box which is attached in a belt. The temperature sensor is located in the inner part of the belt where the metallic encapsulated is in contact with the skin of the animal. To measure the cardiac frequency, we use two electrodes attached with a wrist band to the front legs (the same procedure can be used for a goat). Finally, both electrodes are connected to the circuit using a wire protected by a metal mesh. This system will prevent that the animal can gnaw these connections. Figure 11 shows the sensors placement on the animal. 


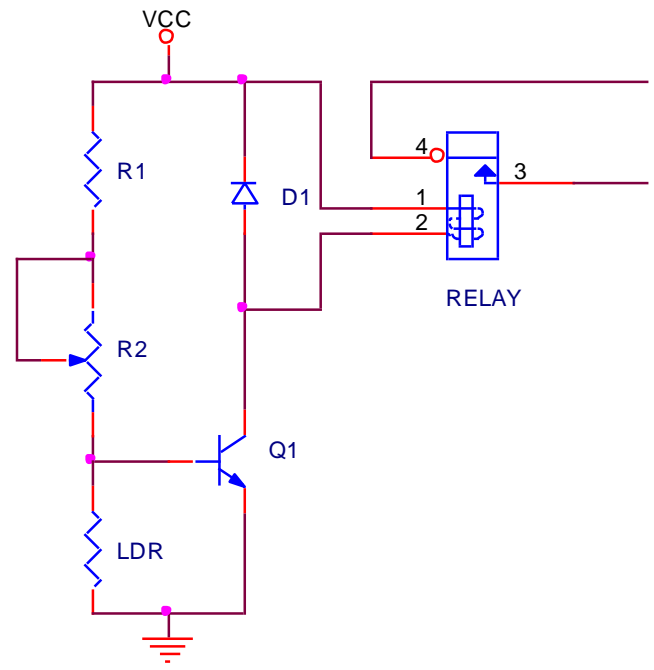

Figure 10. ON/OFF circuit to control the wireless node

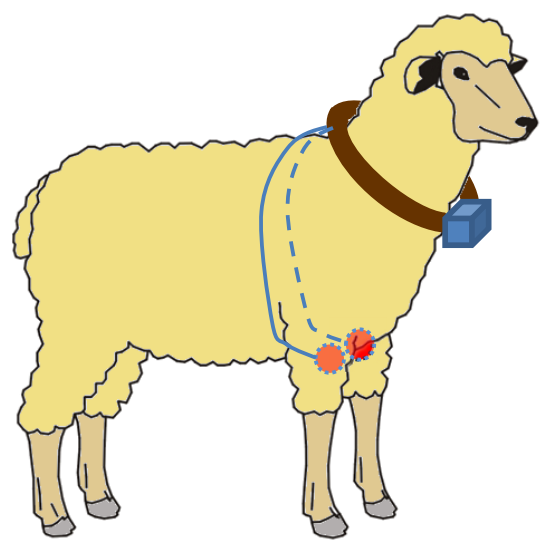

Figure 11. Placement of sensors on the sheep

\section{WIRELESS SENSOR NETWORK}

For our proposal, we use a wireless network of infrastructure type. Each animal (or a percentage of them) wears a wireless node provided with a temperature sensor and an ECG circuit. The set of all nodes forms a WSN which interconnect all nodes through the access point (AP) [21]. Figure 12 shows the structure of the proposed network for repelling wolf attacks.

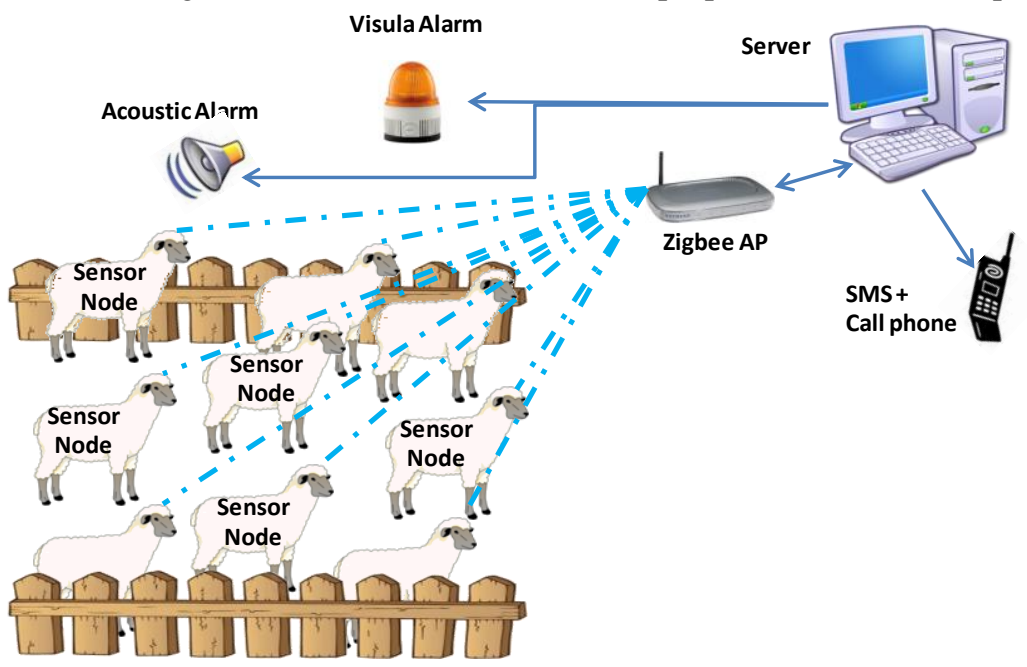

Figure 12. Structure of the wireless network

This section shows the wireless devices used to implement our network and explains the operation of our system. Our design uses ZigBee as a wireless communication protocol. ZigBee is an alliance, without spirit of lucre, of more than 100 companies, where most of them are semiconductors makers. ZigBee and IEEE 802.15.4 are standards-based protocols that provide the network infrastructure required for wireless sensor network applications. IEEE 802.15.4 defines the physical and MAC layers, and ZigBee defines the network and application layers. This technology is focused to create low-rate personal area networks (LR-WPAN). Its low power consumption feature make this protocol ideal for high that require secure communications, but with low data rates at short distances that maximizes the network lifetime [22]. This technology is suitable for low cost communications between nearby nodes without or with very little infrastructure. In order to avoid collisions in sending information, this standard implements the CSMA-CA algorithm (Carrier Sense Multiple Access with Collision Avoidance). This algorithm avoids the problems of hidden nodes, however it does not solve the problem of exposed nodes.

The sensor node is implemented using a Waspmote node [23]. It is designed by Libelium Company. It is able to combine several sensors and actuators by using its programming environment. The programming language is $\mathrm{C}++$ simplified restricted it to the API provided by the manufacturers [24]. Waspmote nodes can be connected by star topology, tree topology or mesh network topology. We have implemented a network in star topology [25]. 
We have selected Ezport ZigBee 101 [26] as AP. It works as a gateway between the ZigBee networks which connect our nodes and the IP network where our server is connected and can offer Internet connection to the rest of the network. This AP can be used for distances up to 100 meters, which is enough to cover the area of a stable.

Our system uses the signals from nodes to determine if there is a situation of collective stress or not. These episodes can be generated for natural reasons or due to the presence of predators or intruders. When an alarm from an animal is generated, the AP communicates this situation to the server. When our system detects 5 alarms from 5 animals within a maximum period of 5 minutes, the server will consider this situation as a situation of collective stress. The first stage in alarm process is focused on frighten and scare off potential predators or intruders with acoustic and visual alarms. The server is the responsible to activate them. In addition, the server sends a text message, warning of the time of the alarm, followed by an automatic call, to the farm owner.

\section{SYSTEM ALGORITHMS}

This section shows the communication protocol and the algorithms designed to take decisions based on the information received from the sensor nodes. In addition, we are going show the procedure to generate alarms when a situation of collective stress is detected.

The operation of our wireless sensor network is based mainly on a smart decision algorithm based on the signals received from the flock of sheep or goats. The algorithm goal is to assess whether it is producing a collective stress situation by the presence of a predator. Because our nodes only work during the night hours (during the day, sheep and goats are with the shepherd, the energy regulator is the responsible of determining when the node will be working, and when the battery must be recharged.

This section shows the three necessary algorithms for the operation of the wireless sensor network. They help us to determine if the flock is being attacked. We are also going to explain the smart algorithm that analyzes the received data. Using a previous learning phase, the system will be able to define the type of alarm and the type of alert that should be generated.

\subsection{Decision algorithm based on an smart tagging system.}

An animal can register an increase of their vital signs due to multiple causes. However, this does not mean that the animal is being attacked. But, generally, when a wolf or an intruder is near the flock, several animals are altered.

Our system tries to control this behavior by following next procedure. When an animal registers elevated body temperature and heart rate, the system process the event as an individual alarm signal, which is sent to the AP. The AP will send this event to the server responsible of the control of the area and it will generate a message. At this time, a 5 minutes timer is activated. If there are 5 or more messages before the account expires, the system will consider that it is being produced a collective stress situation. The detection of a collective stress situation activates the acoustic and luminous alarms. Simultaneously, the server will send a text message to the farm owner or other people depending of the type of case, which is decided by a smart algorithm.

The system can detect several types of cases. Each situation will generate a different type of alarm, which will advise different persons.

Firstly, a sheep or goat may be altered by natural causes, such as the presence of a female in mating season (alarm level 1). But the animals may be frightened by the presence of thieves (alarm level 2), by the presence of wild animals (alarm level 3) or by the presence of some kind of natural phenomena, such as a fire, a flood, etc (alarm level 4).

Each type of alarm (from the lowest, level 1, to the highest, level 4), must be addressed. For this reason, when a level 1 alarm is generated, the system will only notify this status to the farm owner. When an alarm of level 2 is generated, the system will warn the farm owner and the police (to try to catch the alleged thief). A level 3 alarm will generate a warning message to the farm owner and to the environmental unit for animal control. Finally, a level 4 alarm means that due to an unusual event, the entire flock is in danger. So, the alert will be communicated to the owner and to the emergency services.

Figure 13 shows the decision algorithm which will control the generation of alarms.

For the proper operation of our intelligent algorithm, we must perform a training system for alarm tagging. In order to achieve this goal, we need to establish a period of manual alarms tagging. This allows us to create an initial alarm database. This database will provide the flock behavior patterns.

When the system detects a collective stress situation ( 5 o more messages received in less than 5 minutes), the time values and messages are stored. Then, the algorithm will analyze each received data which will be compared with the data from the database (the initial data comes from the training phase). After determining the priority of the alarm, the system will perform, on the one hand, an alarm tagging and then, it will be save in the database, and, on the other hand, the communication and the alarm generation (Visual alarm, acoustic alarm and GPRS communication).

The intelligent algorithm, allows the WSN reporting the status of the animals. In addition, when there is a threat, the system will generate several deterrents (visual and acoustic alarms) that may scare the threats, while the owner is going to the farm. 


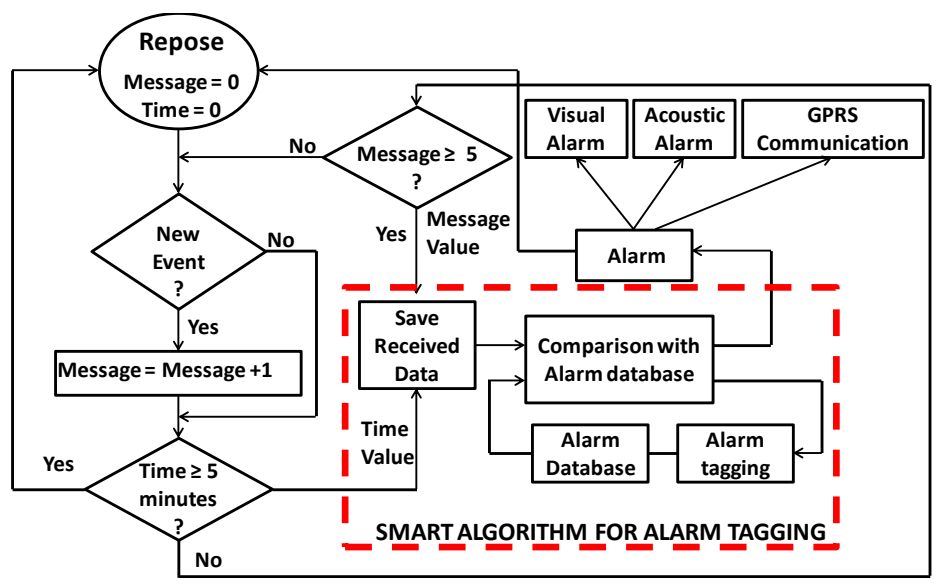

Figure 13. Decision algorithm

\subsection{Energy regulator control Algorithm}

One of the most important issues to be considered in WSNs is to have as low as possible power consumption. This will prolong the network lifetime and facilitate to maintain more stable communications [27].

In order to prolong devices lifetime, we use an energy regulator algorithm which is able to control the periods when a node will work and when the battery needs to be recharged.

Figure 14 shows the control algorithm to control the energy regulator. First, we should detect if it is night or not. During the daytime, the system must check the battery status. If the charge level is below $100 \%$, the battery should be recharged through the solar panel. Otherwise, the system will remain in idle mode and it will turn off the node. When night falls, the energy regulator control activates the relay that controls the battery connection to the sensor node, enabling wireless link with the AP.

\subsection{Communication Protocol}

When a sensor node wakes up, it attempts to communicate with the AP. In order to do this, the node scans the wireless networks that are within range. The AP configured to let the access to the sensor nodes will reply with an ACK message. Each node will request the authentication to the AP which will respond with a confirmation (if the node is registered).

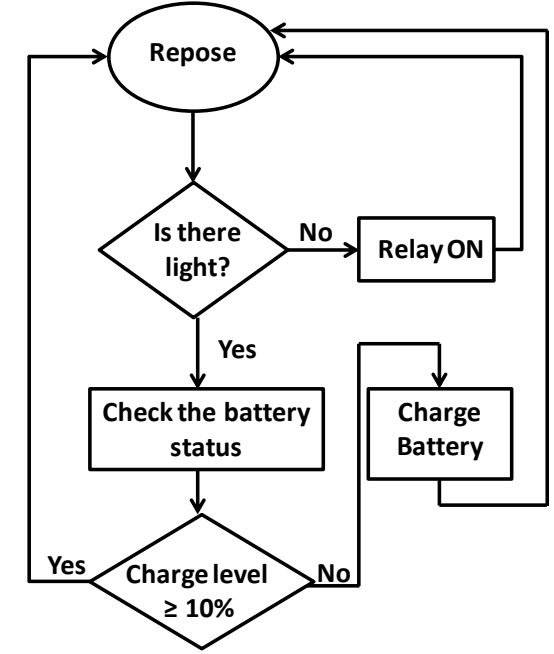

Figure 14. Algorithm to control the energy regulator

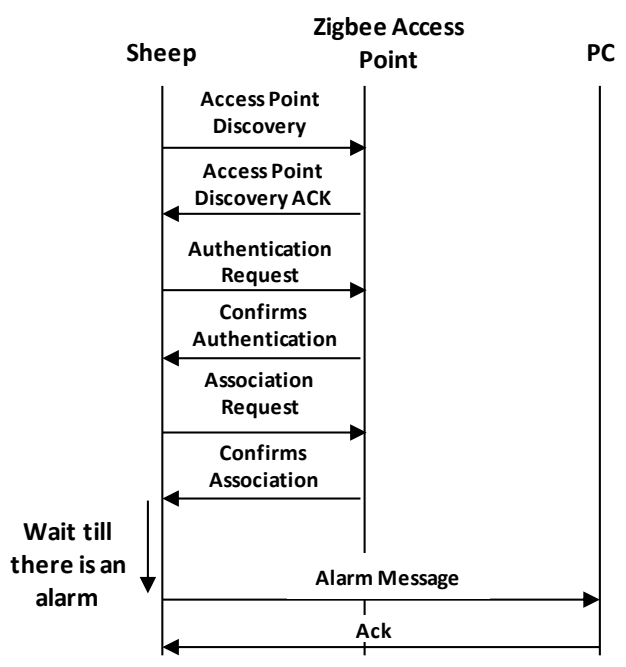

Figure 15. Communication Protocol

Finally, the node of each sheep or goat will be associated with the AP. From that moment, the AP will await the arrival of any alarm message. Figure 15 shows the message flow procedure between a sensor node and the AP. 


\section{Performance Study}

This section simulates the decision algorithm that generates the alarms. We also show real measurements from a real environment using Zigbee wireless technology.

Figure 16 shows the simulation results of our decision algorithm. As we can observe, an alarm is generated individually at 50 seconds causing the first signal message. After 5 minutes no more alarms are registered and consequently, the value of the alarm signal returns to 0. From the second 390, different individual alarms are generated, so the message value increases. When this value is equal or higher than 5 , the alarm is activated.

In order to show the performance of our Zigbee network, we have connected a node Zigbee and Zigbee AP. This allows us to study the individual network performance due to the operation of a single node placed in single sheep or goat.

Figure 17 shows the bandwidth in bits per second consumed in the network due to the communication protocol. In 15th second, the node sends a broadcast message to discover the reachable APs. Immediately, it receives an ACK message from the AP. After this reception, the node authenticates with the AP using the password that was previously configured. The node receives the confirmation of this authentication (which is received approximately on the 18th second). Finally, the node sends an alarm message in the 31 st second and receives the ACK message.

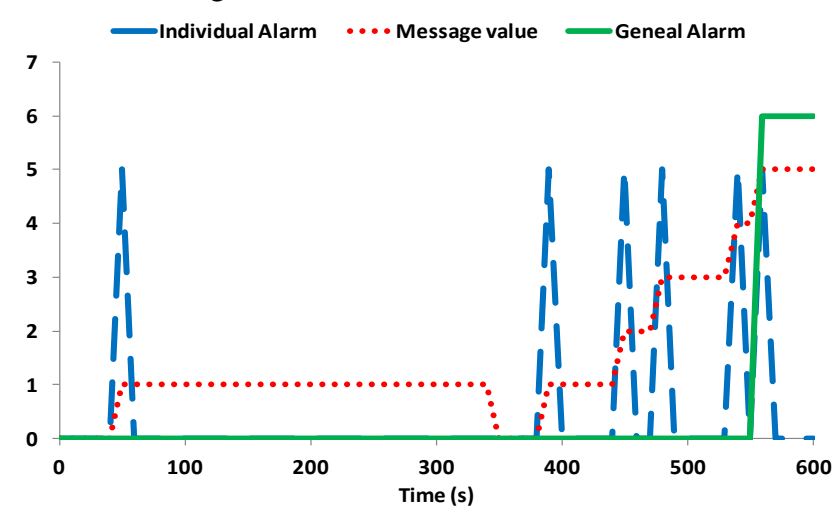

Figure 16. Simulation of the decision algorithm

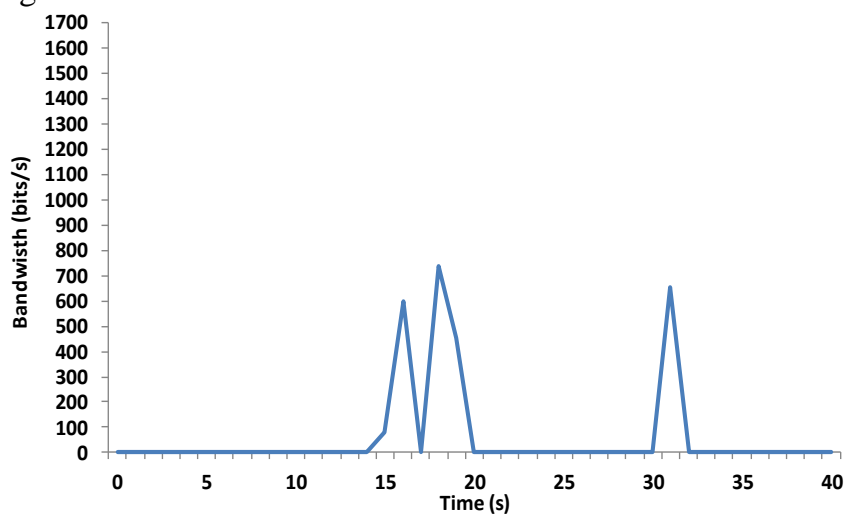

Figure 17. Consumed bandwidth

Figure 18 shows the bytes sent by the sensor node during the above described communication process. The moment with greater registered number of bytes is when the node tries to associate with the AP. We noticed that there is another peak of number of bytes when the node sends the last alarm (last peak of the graph).

Figure 19 shows the responses of AP to messages sent by the node according to the described communication protocol. We can see that there is more number of bytes sent during the sensor nodes registration process, so the system does not consume too much bandwidth once all sensor nodes have been registered.

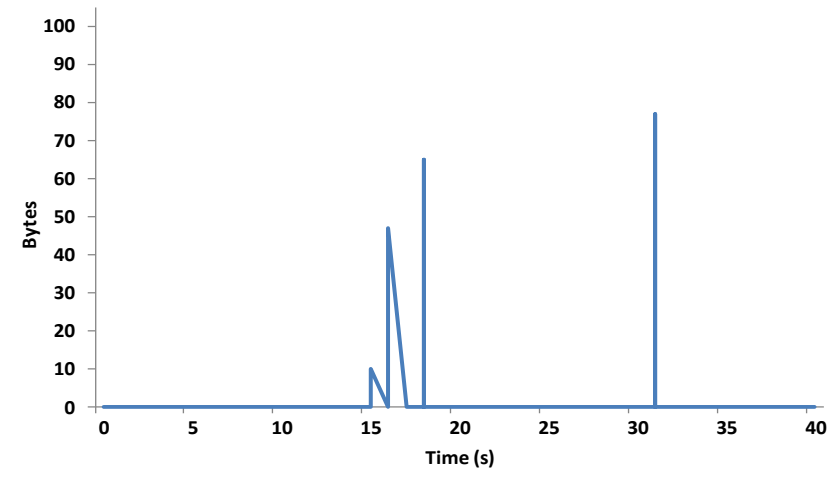

Figure 18. Bytes sent by the node

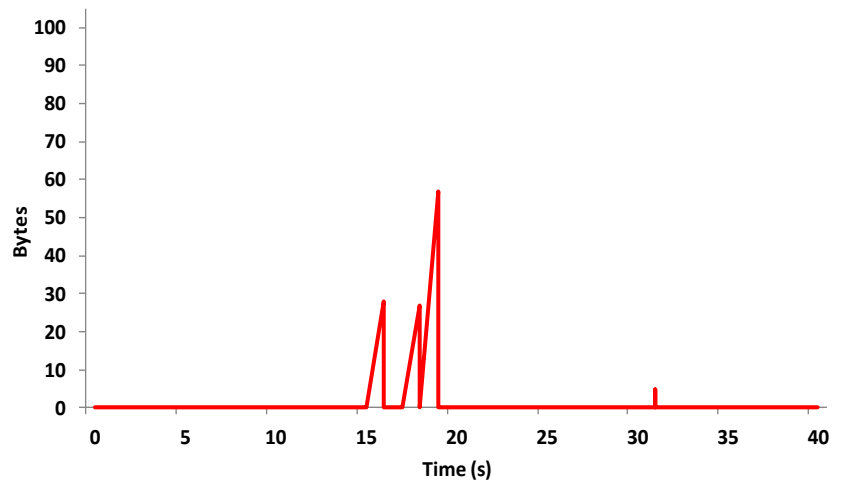

Figure 19. Bytes sent by the AP.

\section{Conclusion}

Economical losses in livestock sector due to wolf attacks or theft are very frequent. In this paper, we have presented the design and development of a WSN to prevent these facts. Our wireless system is based on the IEEE 802.15.4 standard.

It is capable of monitoring the vital constants of animals and decides, based on these values if there is an episode of collective stress in the sheep and goats flock. To define the status of our flock, our smart system uses the variations of heart rates and corporal temperature of the animals. Taking into account these values, the system can detect the attack of predators or 
thieves. Our system controls the alarm system to scare predators and to generate the appropriate alerts to the owner of the livestock and to other people.

The sensors which measure the frequency heart and corporal temperature are placed on a belt that does not disturb the animal to walk.

Due to the energy limitations, our nodes are endowed by an intelligent energy saving system. This system is based on recharges through a solar panel and a node control unit which limit its operation only during the night. For this reason, our system can be considered totally self-sufficient.

This smart system can be considered as a tool to prevent the loss of the cattle, increasing production and profits from livestock facilities. This system can be implemented in any type of livestock facilities and can be used to alleviate the economic losses.

As a future work, we want to check the operation of our system in a real environment. To this, we would like to avoid the wire connections in ECG electrodes. The best system could be a wireless connection between electrodes and collar. So we will deploy a wireless body area sensor network to interconnect them. We would also include a video surveillance system connected to the server using IEEE 802.11 technology. In this way, we will be able to identify possible intruders.

Finally, we would like to extend this system to other kinds of animals which are also affected by attacks, generating high losses.

\section{REFERENCES}

[1] Wark T, Jurdak R, "Energy management for wireless sensor networks", App. Num.: US2010000265800. Published date: 2012-0927. United States of America

[2] Bri D, Garcia M, Lloret J, Dini P, "Real deployments of wireless sensor networks", In proceedings of the third International Conference on Sensor Technologies and Applications, SENSORCOMM'09. Athens/Glyfada (Greece), June 18-23, 2009.

[3] Kim J, Lim J H, Kim K T, Jang B. T., Choi J. D., Kim D. H., Yoo J., Sung K. B., Jang J. A.” Method for wireless communication in wireless sensor network environment", ", App. Num.: US2009012569439, Published date: 2013-06-04. United States of America

[4] Sendra S., Lloret J., Garcia M., Toledo J.F., "Power Saving and Energy Optimization Techniques for Wireless Sensor Neworks", Journal of Communications, 2011: 6 (6); 439-459

[5] ASAJA (Asociación Agraria de Jovenes Agricultores), Online press article (September 4, 2012), "ASAJA Castilla y León apunta que el lobo avanza a "su libre albedrío" sin que el Plan de Gestión consiga sus objetivos de hacerlo compatible con la ganadería". Available

http://www.asaja.com/publicaciones/asaja_castilla_y_leon_apunta_que_el_lobo_avanza_a_\%E2\%80\%9Csu_libre_albedrio\%E2\%80 \%9D_sin_que_el_plan_de_gestion_consiga_sus_objetivos_de_hacerlo_compatible_con_la_ganaderia_738. [Last access 28/08/2013]..

[6] Principado de Asturias Government. Online press article (October 9, 2012): "El principado propone autorizar controles poblacionales del lobo hasta finales de 2013". Gobierno del Principado de Asturias. Available at: http://www.asturias.es/portal/site/webasturias/menuitem.6282925f26d862bcbc2b3510f2300030/?vgnextoid=f1cce3d1ad74a310VgnV CM10000097030a0aRCRD\&i18n.http.lang=es [Last access 28/08/2013].

[7] Ministère de l'Ecologie, du Développement Durable, des Transports et du Logement; Ministère de l'Agriculture, de l'Alimentation, de la Pêche, de la Ruralité et de l'Aménagement du Territoire.Wolf in France, in the french context of and traditional livestock farming. Available at: http://www.rdbrmc-travaux.com/loup/spip.php?rubrique90 [Last access 28/08/2013]..

[8] Llario F., Sendra S., Parra L., Lloret J.," Detection and Protection of the Attacks to the Sheep and Goats Using an Intelligent Wireless Sensor Network", In proceedings of IEEE International Conference on Communications (ICC 2013) - SCPA 2013, June 9-13,2013, Budapest (Hungary)

[9] Vellidis, G., V. Garrick, S. Pocknee, C. Perry, C. Kvien, M. Tucker. How wireless will change agriculture. In: Stafford, J.V. (Ed.), Precision Agriculture '07 - Proceedings of the Sixth European Conference on Precision Agriculture (6ECPA), Skiathos, Greece, 2007; pp. 57-67.

[10] Huircan J.I., Bustos J., Muñoz C., Vivallo G., Barrigaz V., Donoso G. and, Toneatti M., "TICs y Ganadería: Manejo Electrónico de Ganado". In proceedings of international workshop, 3rd Encuentro Informática y Gestión (EIG2009), Temuco (Chile), Decembre 3-4, 2009.

[11] Lloret J., Bosch I., Sendra S., and Serrano A. (2011). “A wireless Sensor Network that use Image Processing for Vineyard Monitoring. In Sensors 2011:11 (6); 6165-6196.

[12] Kwong, K. H., Wu, T. T., Goh, H. G., Stephen, B., Gilroy, M., Michie, C., \& Andonovic, I.. Wireless sensor networks in agriculture: Cattle monitoring for farming industries. In Progress In Electromagnetics Research Symposium 2009: 5 (1); 31-35.

[13] Arce A.I.C., Tech A.R.B., Silva A.C.S. and, Costa E.J.X., "Wireless sensor networks for bovine herd monitoring". Archivos de zootecnia 2008: 58 (222), 253-263.

[14] Hwang J. and, Yoe H., "Study of the Ubiquitous Hog Farm System Using Wireless Sensor Networks for Environmental Monitoring and Facilities Control". Sensors 2010: 10 (12); 10752-10777.

[15] Quiza J., Castelblanco T. and, González D., "Sistema Web de Identificación de Ganado Bovino y Monitoreo en Tiempo Real de su Temperatura y Frecuencia Cardiaca Usando Redes de Sensores Inalámbricos". ENGI Revista Electrónica De La Facultad De Ingeniería 2012: 1(1).

[16] Hicks L.C., Hicks W.S., Bucklin R.A., Shearer J.K., Bray D.R., Soto P. and, Carvalho V., "Comparison of Methods of Measuring Deep Body Temperatures of Dairy Cows". ASAE Proceeding of the 26th International Symposium, May 21-23, 2001, Louisville, Kentucku, (USA). 2001; Núm. 701P0201: 432-438. 
[17] Martinez A, Schoenig S., Andresen D. and, Warren S., "Ingestible Pill for Heart Rate and Core Temperature Measurement in Cattle". In proceedings of 28th Annual International Conference of the IEEE Engineering in Medicine and Biology Society (EMBS'06), August 30 - September 3, New York City, New York ( USA), 2006; 3190-3193.

[18] Nagll L., Schmitzl R., Warren S., Hildreth T.S., Erickson H. and, Andresen D., "Wearable Sensor System for Wireless State-of-Health Determination in Cattle". In proceeding on the 25th Annual International Conference of the IEEE Emgimeers in Medicine and Biology Society, September 17-21. Cancun, (Mexico), 2003; 3012-3015.

[19] EFE Agency. Online press article (August 11, 2012), "Que viene el lobo: las ovejas ya avisan a través de mensajes de texto". Available at: http://www.20minutos.es/noticia/1560855/0/ovejas/sms/lobo/ [Last access 28/08/2013].

[20] Temperature sensor P0K1.516.7W.B.010 features. Available at Farnell web site http://www.farnell.com/datasheets/1644002.pdf [Last access 28/08/2013].

[21] Allard-Jacquin P, Coutelou O, Thonet G, "Communication gateway between wireless communication networks", App. Num.: US2007000747579, . Published date: 2012-10-02. United States of America

[22] Yassien M.O.B., Salayma M. Kh., Mardini W. E. and, Khamayseh Y. M., "Fibonacci Backoff Algorithm for IEEE 802.15.4/ZigBee", Network Protocols and Algorithms 2012: 4 (3); 62-78.

[23] Datasheet Waspmote. Available at Libelium Comunicaciones Distribuidas S.L. web site: http://www.libelium.com/documentation/waspmote/waspmote-datasheet_eng.pdf . [Last access 28/08/2013].

[24] Martín C., Villa D., Aceña O., Villanueva F.J., Moyaand F., López J.C., "Plataforma de alto nivel para el desarrollo de sensores y actuadores de bajo coste en entornos inteligentes". Laboratorio ARCO, Escuela Superior de Informática, Universidad de Castilla-La Mancha. Available at: https://arco.esi.uclm.es/public/papers/2011-Ei3-cleto.martin.pdf. [Last access 28/08/2013].

[25] Liang W, Yu H, Zhang X, Yang M, Xu W, Wang J, Zeng P, Yang Z, “Communication method for mesh and star topology structure wireless sensor network", App. Num.: EP2009000832920, Published date: 2012-10-31, European Patent Office (EPO)

[26] Datasheet AP Ezport-101. Available at Passport Networks inc. Web site: http://passportnetworks.com/download/EZport_um.pdf. [Last access 27/12/2012].

[27] Segal M., "Improving lifetime of wireless sensor networks", Network Protocols and Algorithms 2009; 1(2); 48-60. 